
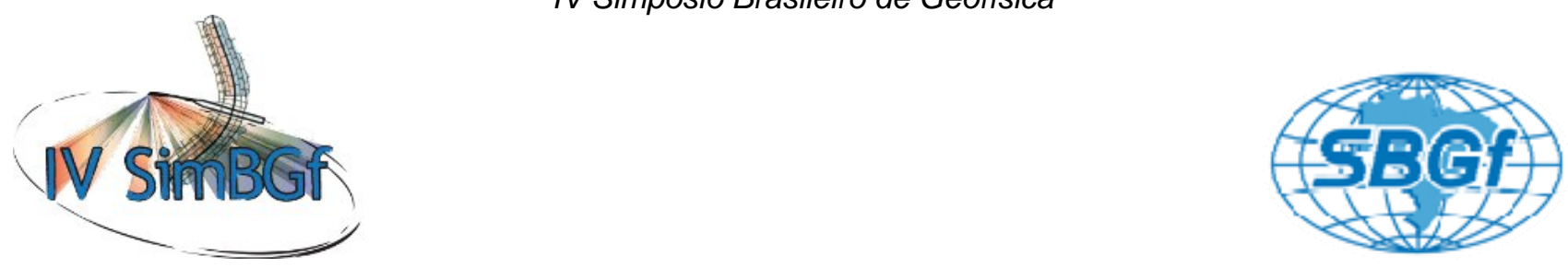

\title{
Estimativas do tensor de impedância utilizando métodos de processamento robusto
} Leonardo Miquelutti, Emin Ulugergerli, Sergio Fontes, Deivid Nascimento, Observatório Nacional.

Copyright 2010, SBGf - Sociedade Brasileira de Geofísica

Este texto foi preparado para a apresentação no IV Simpósio Brasileiro de Geofísica, Brasília, 14 a 17 de novembro de 2010. Seu conteúdo foi revisado pelo Comitê Técnico do IV SimBGf, mas não necessariamente representa a opinião da SBGf ou de seus associados. É proibida a reprodução total ou parcial deste material para propósitos comerciais sem prévia autorização da SBGf.

\section{Resumo}

Um conjunto de dados magnetotelúricos foi processado de diferentes formas, no domínio da frequência, visando comparar resultados e sugerir novos procedimentos que venham produzir resultados mais suaves. A obtenção do tensor de impedância e, conseqüentemente, resistividade e fase aparentes foi realizada de quatro maneiras diferentes, sendo a primeira deles envolvendo o processamento por mínimos quadrados clássico, enquanto nos outros três procedimentos empregou-se processamento robusto.

\section{Introdução}

O método magnetotelúrico (MT) visa a obtenção do tensor de impedância através do processamento de séries temporais de determinadas componentes dos campos elétrico e magnético terrestres, gerados naturalmente. Através do tensor de impedância, é possível obter, por exemplo, gráficos de resistividade e fase aparentes $x$ freqüência, dos quais se extrai a resistividade $x$ profundidade do meio sendo investigado.

O processamento dos dados pode ser feito de várias maneiras no domínio da frequência, dentre as quais se destacam o processamento clássico, a referência remota e o processamento robusto (Gamble et al., 1979; Jones et al., 1999; Egbert, 2002; Chave and Thompson, 2004, etc.). O processamento clássico consiste na utilização dos métodos dos mínimos quadrados. A referência remota consiste na utilização de componentes dos campos elétrico ou magnético de outra estação, de forma a minimizar o erro, admitido como não-correlacionado entre as duas estações em questão. O processamento robusto consiste na utilização de técnicas robustas de processamento, de forma a excluir os dados cujos erros são relativamente grandes (outliers), e utilizar apenas os dados com boa qualidade na geração de resultados.

Neste trabalho, um programa desenvolvido em linguagem C, baseado no software MTR93, da EMI, faz a comparação entre o processamento clássico e três formas distintas de processamento robusto, de forma que possibilita a comparação entre essas diversas técnicas.

\section{Metodologia/ Problema Investigado}

No método $\mathrm{MT}$, o tensor de impedância $Z$ está relacionado com os campos elétrico $E$ e magnético $H$, considerando o problema como bidimensional (2-D), através da seguinte relação no domínio da frequência:

$$
\left(\begin{array}{l}
E_{x y} \\
E_{y}^{v}
\end{array}\right)=\left(\begin{array}{ll}
Z_{x w} & Z_{x y} \\
Z_{y x} & Z_{y y}
\end{array}\right)\left(\begin{array}{l}
\frac{B_{x}}{\mu_{0}} \\
\frac{B_{y}}{k_{0}}
\end{array}\right)
$$

Medem-se, em geral, três componentes do campo magnético terrestre e duas componentes do campo elétrico, durante determinado tempo, de modo que os dados obtidos são séries temporais, cujo processamento resulta nos valores do tensor de impedância.

Através do tensor de impedância, é possível estimar a resistividade aparente $\rho_{a}$ e a fase $\Phi$, em situações (2-D):

$$
\begin{aligned}
& \beta_{a, j f}(\omega)=\frac{\left|z_{i f}(\omega)\right|^{2}}{\mu_{0} \omega} \\
& \phi_{i j}=\tan ^{-1}\left(\frac{\operatorname{Im}\left\{z_{i j}\right\}}{\operatorname{Re}\left(z_{i j}\right)}\right)
\end{aligned}
$$

As componentes do tensor de impedância são obtidas através de crosspower e autopower (potências cruzadas e auto-potências), calculadas no domínio da freqüência. No nosso caso, Crosspowers são produtos de diferentes componentes do campo eletromagnético da Terra para uma mesma freqüência, por exemplo, $\mathrm{E}_{x} \mathrm{H}_{x}$, enquanto autopowers é o produto de uma componente do campo por ela mesma, por exemplo, $\mathrm{H}_{y} \mathrm{H}_{y}$. Para exemplo de ilustração, a componente $Z_{x y}$ é mostrada abaixo:

$$
z_{W y}=\frac{\left\{E_{w} H_{w}^{*}\right)\left(H_{y} H_{y}^{*}\right)-\left(E_{W}^{*} H_{y}^{*}\right)\left(H_{y} H_{W}^{*}\right)}{\left(H_{w} H_{W}^{*}\right)\left(H_{y} H_{y}^{*}\right)-\left(H_{W} H_{y}^{*}\right)\left(H_{y} H_{W}^{*}\right)}
$$

O processamento clássico calcula os valores de cross e autopowers através do Método dos Mínimos Quadrados, uma vez que, para cada freqüência angular $\omega$, há $n$ valores de cada cross ou autopower, $n=100$ no nosso exemplo. O processamento robusto não é um método rigorosamente definido, com diferentes técnicas podendo ser enquadradas como processamento robusto. Três formas foram testadas, a fim de se comparar os resultados com o processamento clássico. 
- A primeira consiste na organização dos valores de cada cross ou autopower em ordem crescente, e na escolha do valor da mediana da magnitude para representação do valor final.

- A segunda consiste no cálculo de uma média da magnitude de cada componente e o erro de cada uma; após isso é calculada a variância e excluemse os pontos cujos erros eram maiores que o dobro da variância. Faz-se uma média ponderada dos valores restantes (cujo peso é o inverso do quadrado do erro) e é atribuído como resultado o valor de magnitude que mais se aproxima dessa média.

- A terceira consiste no tratamento de forma separada dos valores reais e imaginários de cada cross ou autopower, exclusão de pontos cujos erros eram maiores que duas vezes a variância, cálculo de média ponderada dos valores restantes (cujo peso é o inverso do quadrado do erro) e é atribuído como resultado o valor encontrado.

\section{Resultados}

Os resultados para os três métodos descritos se encontram mostrados abaixo. Cada conjunto é composto por um gráfico de "resistividade aparente $x$ período" $\mathrm{e}$ outro de "fase aparente $x$ período".

O método 1 resultou em uma curva cujos valores de resistividade aparente para altos períodos estão completamente incoerentes.
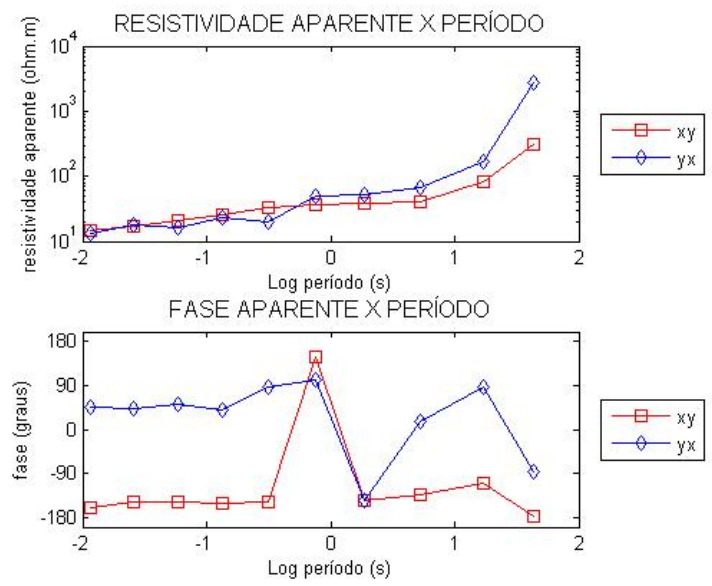

Figura 1 - Resultados obtidos utilizando o método 1

O método 2 apresentou uma curva problemática para altos períodos novamente, principalmente na direção yx da resistividade aparente. $O$ gráfico de fase aparente mostra uma curva suave para a direção xy.

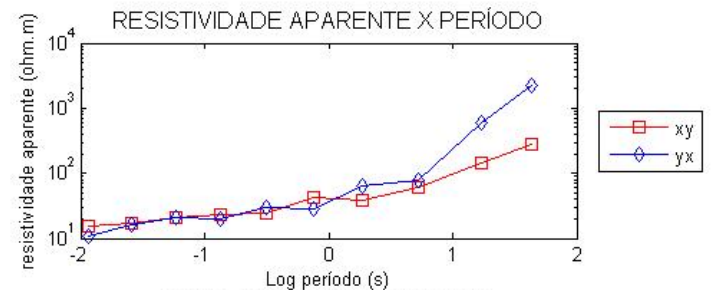

FASE APARENTE XPERIODO

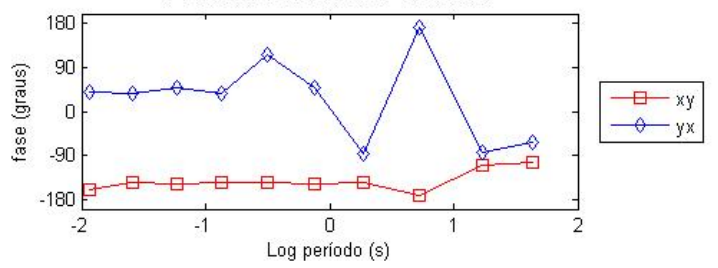

Figura 2 - Resultados obtidos utilizando o método 2

O método 3 apresentou valores mais reais de resistividade aparente em ambas direções, além das curvas de fase aparentes estarem bem-comportadas.
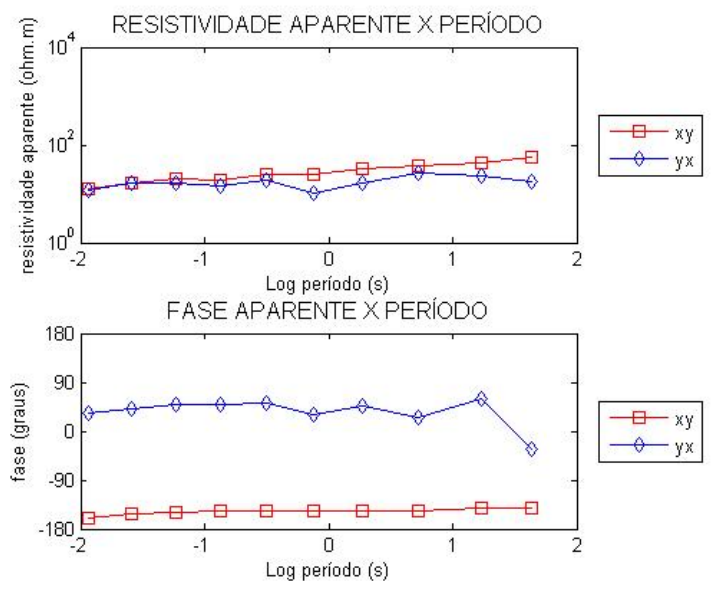

Figura 3 - Resultados obtidos utilizando o método 3

\section{Discussão e Conclusões}

No método 1 , a seleção de apenas um ponto (a mediana) como representativo de todas as medidas se mostrou ineficiente, resultando em valores de resistividade aparente incompatíveis com a realidade.

No método 2, no qual havia um pré-descarte de valores de cross e autopowers, os resultados, apesar de não representarem uma realidade geológica, apresentaram uma melhora em relação ao primeiro método.

No método 3, no qual houve um tratamento matemático dos valores de cross e autopowers (média ponderada), nota-se que as curvas de resistividade aparentes se restringem a valores mais realísticos. A curva na direção xy se comporta de forma suave, como prevê a teoria do 
método MT, assim como as curvas de fase aparente, também muito bem comportadas.

Os métodos 1 e 2, nos quais não houve um tratamento matemático dos valores de cross e autopowers, e sim a escolha de um dos $n$ valores como representante de todos, os resultados não foram melhores que o resultado do processamento clássico, mostrado na figura 4 abaixo.
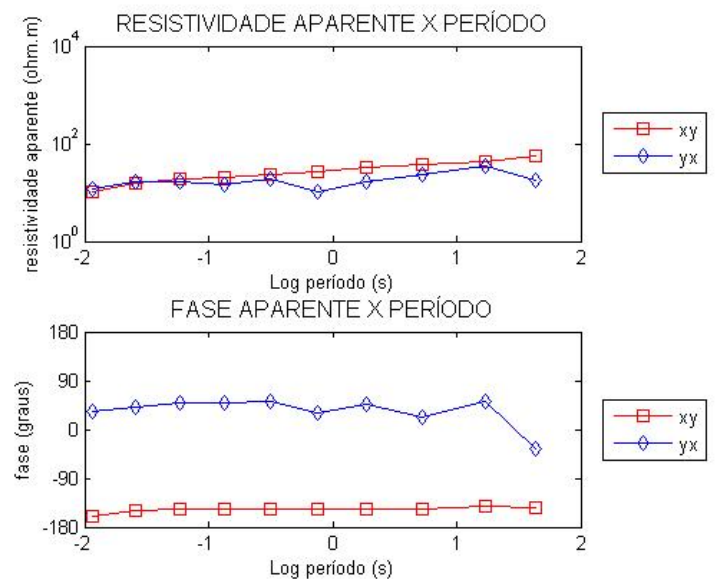

Figura 4 - Resultados obtidos utilizando o método clássico de processamento.

O método 3 , no qual houve um tratamento matemático dos pontos, mostrou um resultado ligeiramente semelhante ao método clássico, sendo sua eficiência inconclusiva até o momento.

Propostas de novos procedimentos se encontram em andamento, e mais métodos propostos, de modo que se possa encontrar uma técnica que resulte em resultados de resistividade e fase aparentes melhores que os obtidos via processamento clássico.

\section{Agradecimentos}

L.M e D.N agradecem a CAPES pela Bolsa de Estudos.

\section{Referências}

Chave, A. D., Thomson D. J., 2004, Bounded influence magnetotelluric response function estimation, Geophyhs. Journal International, 157: 988-1006.

Egbert, G.D., 2002. Processing and interpretation of Electromagnetic induction array data, Surveys in Geophysics 23: 207-249.

Gamble, T.D., Goubau, W.M. and Clark, J., 1979. Magnetotellurics with a remote magnetic reference. Geophysics, vol 44, 53-68.
Jones, A.G., Chave, A. D., Egbert, Audi, D. and G.D. Bahr, K., 1989. A Comparison of techniqyes for magnetotelluric response function estimation. JGR, vol 94, B10, 14.201-14.213. 